\title{
Pattern Identification for State Prediction in Dynamic Data Streams
}

\author{
Shirin Enshaeifar, Seyed Amir Hoseinitabatabaei, Alireza Ahrabian, and Payam Barnaghi \\ Institute for Communication Systems, Department of Electrical and Electronic Engineering, \\ University of Surrey, GU2 7XH, UK. \\ email: \{s.enshaeifar, a.taba, a.ahrabian, p.barnaghi\}@surrey.ac.uk
}

\begin{abstract}
This work proposes a pattern identification and online prediction algorithm for processing Internet of Things (IoT) time-series data. This is achieved by first proposing a new data aggregation and datadriven discretisation method that does not require data segment normalisation. We apply a dictionary based algorithm in order to identify patterns of interest along with prediction of the next pattern. The performance of the proposed method is evaluated using synthetic and real-world datasets. The evaluations results shows that our system is able to identify the patterns by up to $85 \%$ accuracy which is $16.5 \%$ higher than a baseline using the Symbolic Aggregation Approximation (SAX) method.
\end{abstract}

\section{Introduction}

Recent advancements in technology and the wide availability of hardware and software products provide a unique opportunity to stay connected anywhere, anytime and being able to integrate data from almost any physical object into the Internet or what is called as the Internet of Things (IoT). Furthermore, recent advancements in the design of IoT led has to the development of smart systems, such as smart homes and smart cities [1].

In general, efficient access and analysis of big data generated from IoT infrastructures are highly dependent on size and quality, latency, availability, reliability, and continuity of the data and services [2]. In this regard, several works have focused on affixing enriched meta-data to enhance semantic interoperability and to deliver machine-readable (and potentially machineinterpretable) features of sensory data [3]-[7].

Despite the successful application of semantic interoperability in IoT data acquisition, effective analysis and visualisation of the real-time IoT data (the socalled 'data analytics') is still a challenging task for many of the IoT infrastructures. To this point, some private organisations [8] and academic institutions [9][12] have been focusing on the development of data analytics tools for big data which could be potential IoT analytics solutions. For instance, [9] introduced a data mining software, called Massive Online Analysis (MOA), which is implemented in Java and provides a collection of machine learning algorithms and tools for evaluation. Similarly, [11] established the initial implementation of the Knowledge Acquisition Toolkit (KAT) which is developed in Python and it includes a set of pre-processing, data abstraction and annotation techniques. Furthermore, [12] proposed the extension of KAT tool as a web service (KAT-WS) which enables the data consumers to have access to a range of algorithms for the analysis of sensor data.

The majority of the IoT data analytics toolkits, including above-mentioned, focus on the analysis algorithms to extract meaningful information from historical sensory data. However, enabling proactive decision making for emergency situations is neglected in most of the data anlytics tools, e.g. [10], [12]. The proactive decision making could be performed based on the prediction of the future state of the sensory data. For example, predicting road traffic states could improve road congestion, or predicting energy patterns could be used develop energy saving mechanisms. In this regard, we have developed an algorithm to predict the next state of the real-time data.

Most of the prediction algorithms found in literature focus on the estimation of individual values based on the presence of historical data, e.g. financial stock price prediction or wind forecasting in renewable energy [13], [14]. However, individual samples in IoT data often do not represent the complete information in a continuous stream of data. This is why they are generally aggregated and analysed to extract higherlevel information from the raw sensory data. In this paper, we aim to predict the future 'state' of the IoT data, rather than estimating a single value. To this 
end, we first analyse the historical data in order to identify the unknown patterns, given that the historic data was available - similar to conventional prediction algorithms. The state prediction is then performed based on the patterns identified from the historical data, i.e. pattern-based state prediction (PSP). The impact and enhanced performance offered by our approach is illustrated on synthetic data as well as real-world processes.

\section{Pattern-based State Prediction}

To develop a pattern-based state prediction (PSP) algorithm, we first need to identify the patterns from the historical data. We have developed an algorithm to aggregate the data and transform it into a discretised series of symbols. This was inspired by symbolic aggregate approximation (SAX) method, which is one of the most well-known algorithms that transforms time-series data into a series of letters referred to as a word [15]. In the second step, we use these patterns to predict the most probable future state. These steps are described in the following sections.

\subsection{Historical Data: Identification of Un- known Patterns}

To analyse the historical data, the raw sensory data were first passed through a pre-processing step in which some algorithms are applied to clean the data from missing values and to remove the outliers. In the next step, similar to other IoT data anlytics tools, dimensionality reduction algorithms were required to reduce the size and length of the data while maintaining the key features and patterns. Furthermore, to facilitate pattern identification, it was necessary to apply further transformations to obtain a discrete representation. One of the most well-known algorithms for this purpose is SAX which performs the dimensionality reduction and also transforms time-series data into a discretised series of letters referred to as a word [15].

The SAX algorithm has been used as a building block for data aggregation, patterns and outliers detection, clustering, and also classification [16]. Note that SAX is composed of the following steps:

1) Normalisation and piecewise aggregation approximation (PAA): In the first step, the original process $\mathbf{x}$ is normalised so that it has zero mean $\left(\mu_{\mathbf{x}}=0\right)$ and unit standard deviation $\left(\sigma_{\mathbf{x}}=1\right)$. Then, PAA algorithm is applied to divide the normalised data of length $N$ into $n$ equally sized windows by taking the mean of each window:

$$
\begin{aligned}
\mathbf{x}_{N} \quad \stackrel{\text { PAA }}{\longrightarrow} \hat{\mathbf{x}}_{n} & =\left(\hat{x}_{1}, \ldots, \hat{x}_{n}\right) \quad n \leq N \\
\text { where } \quad \hat{x}_{i} & =\frac{n}{N} \sum_{j=N / n(i-1)+1}^{(N / n) i} x_{j}
\end{aligned}
$$

Observe that PAA smooths the data and also reduces the dimensionality from $N$ to $n$. Although PAA is intuitive and simple, it was shown to rival other dimensionality reduction methods such as Fourier transforms and wavelets [15].

2) Discretisation: After applying PAA on the normalised data, a further transformation is required to provide the discrete representation; preferably using symbols with equiprobability. Since the normalised data have a Gaussian distribution, SAX algorithm determines a list of 'breakpoints' that produce equal-size areas under the Gaussian curve, i.e. breakpoints are a sorted list of numbers $\mathbf{B}=b_{1}, \ldots, b_{\beta}$ where $\beta$ is a pre-set parameter defining the number of breakpoints [15].

Once these points are determined, they are used to discretise the PAA output vector. For which, all the PAA values that are smaller than the first breakpoint are mapped to the first symbol ' $\boldsymbol{a}$ ', the values that are larger than the first breakpoint and smaller than the second breakpoint are mapped to the second symbol ' $\boldsymbol{b}$ ', and so on.

$$
\begin{gathered}
\hat{\mathbf{x}}_{n}=\left(\hat{x}_{1}, \ldots, \hat{x}_{n}\right) \quad \stackrel{\text { Discretise }}{\longrightarrow} \hat{\mathbf{s}}_{n}=\hat{s}_{1}, \ldots, \hat{s}_{n} \\
\text { where } \quad \hat{s}_{i}=\operatorname{symbol}(j) \quad \text { iff } \\
b_{j-1}<\hat{x}_{i}<b_{j}
\end{gathered}
$$

Despite the advantages of SAX algorithm, patterns detected via this method could not be used for online state prediction. This was mainly due to the normalisation procedure in which statistics of the original data (mean and standard deviation) were ignored. Accordingly, the state of the incoming test data could not be predicted, as real-time timestamps were not normalised. Therefore, in this paper, we proposed a scale independent representation (SIR) algorithm, in which PAA was applied on the original data (without normalisation) and breakpoints were defined according the range of the PAA output vector.

Note that conventional PAA requires the output length $n$ (or the aggregation window size $\tau=\frac{N}{n}$ ) as a pre-set parameter. However, in this paper, we defined the window size as:

$$
\tau \approx \frac{1}{L_{p}} \times T_{p} \times s p m \times 60
$$

where spm is the number of samples per minute and $T_{p}$ is the 'temporal duration' of patterns of interest 
(hour-based). In addition, we defined $L_{p}$ as an optional parameter to set the desirable 'symbolic length' of patterns, i.e. number of segments used to represent a pattern ${ }^{1}$. For example, if energy data was recorded with $s p m=5$ and the user was interested in 6-hours patterns, the aggregation window for PAA was set to $\tau=\frac{1}{3} \times 6 \times 5 \times 60$.

Once the data was discretised and transformed into the symbolic representation using SIR, it was further analysed to extract the repetitive patterns, called as 'SIR-P' algorithm. Since there was no prior knowledge on the structure of the patterns of interest, we extracted all the existing patterns and identified their frequencies of occurrence; these frequencies were then used to detect the common patterns for prediction of the future states. This technique was inspired by the Lempel-ZivWelch (LZW) algorithm [17] which is a well-known dictionary-based approach for data compression ${ }^{2}$. Note that the minimum length of patterns was considered as 2 , where two consecutive symbols represented a pattern, and its maximum was considered as $n$, meaning that the whole PAA vector represented a single pattern. In this work, we extract all the existing patterns with the maximum length of $L_{p}+1$ symbols. The SIR-P method is summarised in Algorithm 1.

\subsection{Stream Data: Prediction of Future State}

After analysing the historical data and generating the dictionaries, we predicted the future state in real-time given the latest pattern $\hat{\mathbf{s}}$ with length $L_{p}$.

We define a probability scale for all the potential future states, i.e. y. To achieve this, the generated dictionary $\mathbf{D}$ is used to acquire all the existing subpatterns $\mathbf{d}_{i}$ that emerge from $\hat{\mathbf{s}}$ with the following characteristics:

$$
\mathbf{d}_{i}=\hat{s}_{i}, \ldots, \hat{s}_{L_{p}}
$$

where $\hat{s}_{i}$ represents the $i$-th character of $\hat{\mathbf{s}}$ sub-string. This way, all the possible subsets of $\hat{\mathbf{s}}_{T}$ were considered for the prediction procedure. Then, the probability scale for the $j$-th future state i.e. $y_{j}$, is calculated as the average occurrence probability of $y_{j}$ given one of the extracted sub-patterns (e.g. $\mathbf{d}_{i}$ ).

$$
P\left(y_{j}\right)=\frac{1}{L_{p}-1}\left(\sum_{i=1}^{L_{p}-1} F\left[\mathbf{d}_{i}, y_{j}\right] / F\left[\mathbf{d}_{i}\right]\right)
$$

1. In this work, we considered the default value of $L_{p}=3$.

2. The LZW algorithm replaces characters with single codes to generate a dictionary and when a new string of characters is detected, it will be added to the dictionary; comprehensive description of LZW algorithm is available in [17] (Chapter 27) and [18].

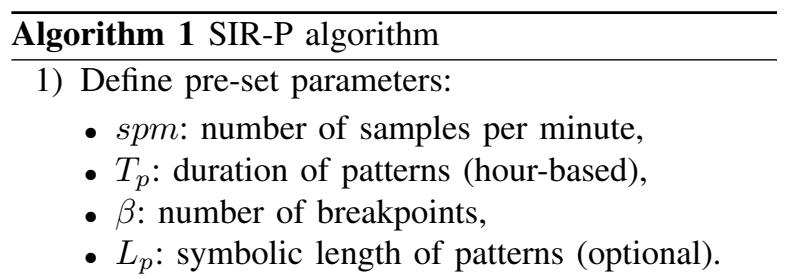

2) Pre-process the original data to clean it from the missing values and outliers.

3) Apply PAA on the clean data to obtain $\hat{\mathbf{x}}$, see Eq. (1).

4) Determine equidistant breakpoints based on the range of PAA

$$
\begin{aligned}
& \mathrm{t}=\frac{\max (\hat{\mathbf{x}})-\min (\hat{\mathbf{x}})}{\beta+1} \\
& \mathbf{B}=\min (\hat{\mathbf{x}})+t: t: \max (\hat{\mathbf{x}})-t .
\end{aligned}
$$

5) Discretise the PAA output based on the selected breakpoints to obtain $\hat{\mathbf{s}}$ using $\beta+1$ symbols.

6) Generate an initial dictionary $\mathbf{D}$ using the existing $\beta+1$ symbols.

7) Find all the possible patterns whose length are less than or equal to $L_{p}+1$ and add them to the dictionary.

8) Define $\mathbf{D}_{f}$ which includes the occurrence frequency of each element in the dictionary ${ }^{3}$.

in which $P$ represents the probability scale and $F$ is the frequency of its sub-string $\left(\left[\mathbf{d}_{i}, \mathbf{y}_{j}\right]\right.$ represents $\mathbf{d}_{i}$ followed by $y_{j}$ ). Accordingly, the potential state with the maximum probability was considered as the predicted value. The overall PSP method is summarised in Algorithm 2.

\section{Evaluation of the SIR-P Algorithm}

To evaluate the performance of the proposed method, it was applied to two different datasets: synthetic data and real-world data such as energy consumption. In order to highlight the advantage of the scale independent method, SIR-P was compared with the conventional SAX algorithm.

\subsection{Evaluation using Synthetic Data}

The first experiment evaluated the prediction accuracy of the proposed method using the synthetic data. To generate the data, autoregressive moving average (ARMA) model was used as to represent the continuous state and sum of sinusoids were used to simulate the patterns of interest. Overall, 50 sets of simulated data were generated to compute the averaged results. Furthermore, white Gaussian noise with high level of signal to noise ratio $(\mathrm{SNR}=0)$ was added to each set. 


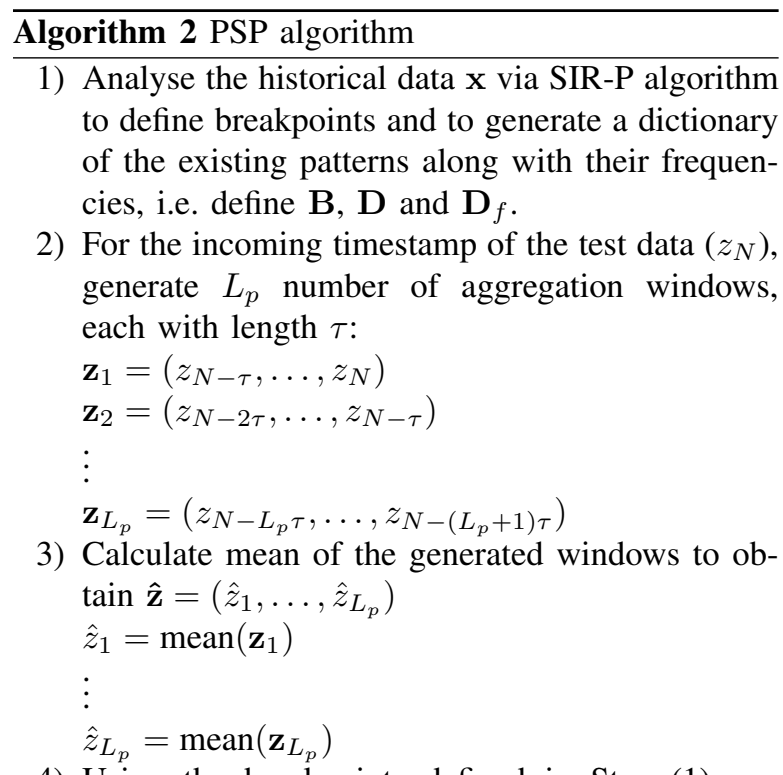

4) Using the breakpoints defined in Step (1), assign a symbol to individual $\hat{z}_{i}$ to generate $\hat{\mathbf{s}}=$ $\left(\hat{s}_{1}, \ldots, \hat{s}_{L_{p}}\right)$.

5) Predict the next state according to the symbol with the maximum probability, see Eq. (5).

The simulated signals were modelled as:

$$
\mathbf{x}=\mathbf{r}+\mathbf{m}_{\Delta t}+\mathbf{e}
$$

where $\mathbf{x}$ is the generated input (e.g. historical data); $\mathbf{r}$ is the ARMA process, $\mathbf{m}_{\Delta t}$ is the pattern of interest over the time range $\Delta t$, and $\mathbf{e}$ is the white Gaussian noise with $\mathrm{SNR}=0$.

In this paper, parameters were defined as $T_{p}=6$, $L_{p}=3, \beta=2$, and $s p m=0.5$; meaning that $\mathbf{x}$ with length $N=5760$ was representing the historical data for eight days. Note that for each set of simulation, a test data $\mathbf{z}$ was generated with length $N=720$ (to represent 24 hours) where it included the similar pattern of interest at a different location, i.e. $\mathbf{m}_{\Delta t_{2}}$.

After generating the input data and selecting the appropriate parameters, SIR-P and SAX were employed to discretise the historical data and construct their corresponding dictionaries. As an illustrative example, Fig. 1 represents an original data and the output of SIR$\mathrm{P}$ algorithm ${ }^{4}$. In the next step, the test data $\mathbf{z}$ was fed to the PSP algorithm. To simulate the real-time scenario, one sample was considered every two minutes (i.e. spm $=0.5$ ) and the next state was predicted for individual timestamps.

4. Note that SIR-P algorithm provides dimnesionality reduction, however, the output shown in Fig. 1 has been reshaped to the original dimension for better visualisation.
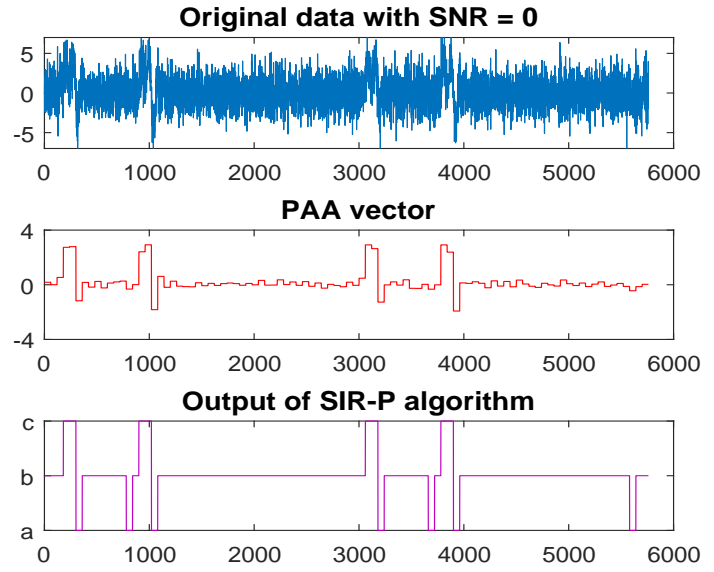

Figure 1: An example of the generated input (top), PAA vector (middle), and the output of SIR-P algorithm (bottom).

To evaluate the performance of the proposed algorithm, the test data was separately discretised, using Algorithm 1, Steps 1-5, to generate the ground truth for our predictions. Accordingly, we calculated the ratio of the number of states which were correctly predicted by our proposed method, i.e. $\rho$. This value was calculated for each simulation set and the final $\rho$ was obtained by averaging over 50 sets of data. Table 1 shows the final prediction accuracies obtained via SIR-P and SAX algorithms.

Table 1: Prediction accuracy

\begin{tabular}{|cc|}
\hline \hline Discretisation method & Final accuracy \\
\hline SIR-P & 0.79 \\
\hline SAX & 0.47 \\
\hline
\end{tabular}

Observe that SIR-P algorithm provided high level of prediction accuracy with $\rho=0.79$; while, using the same parameters and dictionary-based approach, the conventional SAX provided significantly less accurate predictions with $\rho=0.47$. It is worth noting that, these accuracies should be taken as indicative rather than the absolute values that will be achieved. The accuracy of the prediction model varies with the entropy of the time series. In our future work, we aim to investigate the performance of the proposed solution at different levels of entropy.

\subsection{Evaluation using Real-world Data}

The data used in this part were obtained from the environmental sensors installed in some offices at the University of Surrey. The sensor nodes are custom designed boards that were developed at the University 

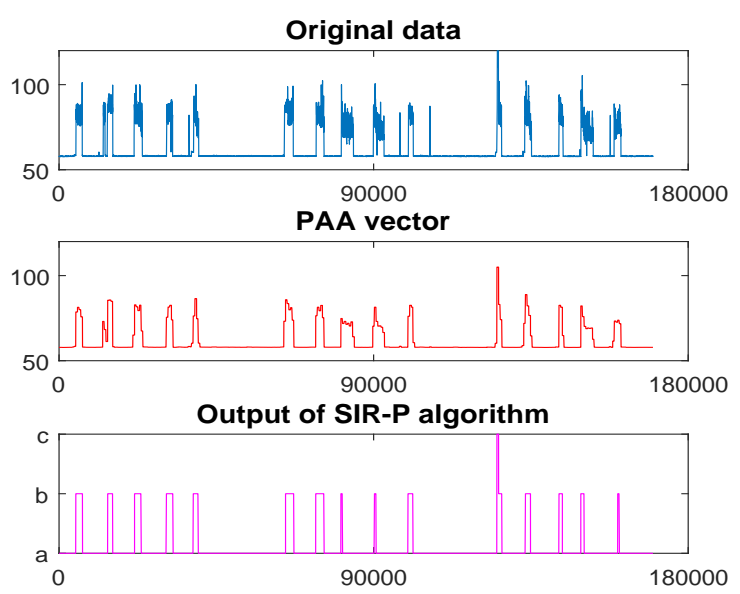

Figure 2: An example of the real-world IoT data (top), which is aggregated via PAA (middle) and then discretised through the SIR-P algorithm (bottom).

of Surrey [19]. These sensors record the environmental data (e.g. light and temperature), noise level, and the energy consumption of the offices. The data is recorded with $s p m=6$, and we defined $L_{p}=3$ and $T_{p}=6$ to be consistent with the synthetic simulations.

For analysis, we considered the energy consumption data recorded over 30 days $^{5}$. The initial 20 days were considered as the historical data, and the following 10 days were used as the test data. Similar to Section 3.1, the historical data was processed using SIR-P, see Fig. 2.

In the next step, the output of SIR-P was used to predict the future state for individual timestamps. For the sake of comparison, prediction was also performed based on the conventional SAX algorithm. To evaluate these methods, a ground truth table was generated by discretising the test data via each algorithm. Accordingly, the overall accuracies were calculated as $\rho=0.85$ and $\rho=0.73$ for SIR-P and SAX, respectively. Compared to the results obtained from the synthetic data, the SAX based algorithm was closer in performance to the proposed method when analysing the real world data. This arises due to the performance of both methods depending on two factors: 1) the complexity of the underlying discretised pattern and 2) the noise levels present in the observed signal. That is, if both the complexity of the discretised pattern as well as the noise level of the observation are low, then both methods will have similar performance. This case is observed in the measured data shown in Fig. 2, where both methods had higher accuracies and their performances were closer.

5. Data is available on request.

\section{Conclusions and Future Work}

In this work we have proposed a new pattern identification and online prediction method. In particular, this work has proposed a scale independent discretisation method, in order to generate a sequence of symbols. In the second part, inspired by the LZW algorithm, a dictionary based learning method was used to identify the existing patterns in the symbols. These patterns were then used to predict the future state using a probability value. We demonstrated the performance of the new approach on both synthetic and real-world simulations, where SIR-P provided significantly higher accuracy than the SAX algorithm.

The future work will focus on the followings. To validate the advantages of the proposed method, we aim to perform further evaluations using a large number of IoT nodes. As mentioned in Section 3.1, the accuracy of the prediction model varies with the entropy of the time series. Therefore, we aim to investigate the performance of the proposed solution at different levels of entropy. Furthermore, in the current PSP algorithm, the number of breakpoint derived from the historical data (i.e. $\beta$ ) was selected as a pre-defined parameter. Therefore, the list of symbols and the generated dictionary $\mathbf{D}$ were fixed, unless the breakpoints were recalculated using a new set of historical data. To resolve this limitation, our future work focuses on the statistical analysis of the historical data to provide adaptive algorithms for online update of these breakpoints and the dictionary.

\section{References}

[1] J. Gubbi, R. Buyya, S. Marusic, and M. Palaniswami, "Internet of Things (IoT): A vision, architectural elements, and future directions," Future generation computer systems, vol. 29, no. 7, pp. 1645-1660.

[2] P. Barnaghi and A. Sheth, "On searching the Internet of Things: Requirements and challenges," IEEE Intelligent Systems, vol. 31, no. 6, pp. 71-75, 2016.

[3] P. Barnaghi, W. Wang, C. Henson, and K. Taylor, "Semantics for the Internet of Things: early progress and back to the future," International Journal on Semantic Web and Information Systems (IJSWIS), vol. 8, no. 1, pp. 1-21, 2012.

[4] D. Pfisterer, K. Romer, D. Bimschas, O. Kleine, R. Mietz, C. Truong, H. Hasemann, A. Kröller, M. Pagel, M. Hauswirth et al., "SPITFIRE: toward a semantic web of things," IEEE Communications Magazine, vol. 49, no. 11, pp. 40-48, 2011. 
[5] D. Guinard, V. Trifa, F. Mattern, and E. Wilde, "From the Internet of Things to the web of things: Resourceoriented architecture and best practices," in Architecting the Internet of Things. Springer, 2011, pp. 97-129.

[6] D. Bandyopadhyay and J. Sen, "Internet of Things: Applications and challenges in technology and standardization," Wireless Personal Communications, vol. 58, no. 1, pp. 49-69, 2011.

[7] Z. Song, A. A. Cárdenas, and R. Masuoka, "Semantic middleware for the Internet of Things," pp. 1-8, 2010.

[8] "IBM Watson: The AI platform for business," https://www.ibm.com/watson/, Accessed: 2017-03-20.

[9] "Massive Online Analysis (MOA): An open source framework for data stream mining," http://moa.cms.waikato.ac.nz/, Accessed: 2017-0320.

[10] M. Hall, E. Frank, G. Holmes, B. Pfahringer, P. Reutemann, and I. H. Witten, "The WEKA data mining software: an update," ACM SIGKDD explorations newsletter, vol. 11, no. 1, pp. 10-18, 2009.

[11] F. Ganz, D. Puschmann, P. Barnaghi, and F. Carrez, "A practical evaluation of information processing and abstraction techniques for the internet of things," IEEE Internet of Things Journal, vol. 2, no. 4, pp. 340-354, 2015.

[12] A. Ahrabian, S. Kolozali, S. Enshaeifar, C. C. Took, and P. Barnaghi, "Stream data analysis as a web service: A case study using iot sensor data," Proceedings of IEEE International Conference on Acoustics, Speech and Signal Processing (ICASSP), 2017.

[13] N. Golyandina, V. Nekrutkin, and A. Zhigljavsky, "Analysis of time series structure: SSA and related techniques."

[14] D. Mandic, S. Javidi, S. Goh, A. Kuh, and K. Aihara, "Complex-valued prediction of wind profile using augmented complex statistics," Renewable Energy, vol. 34, no. 1, pp. 196-201, 2009.

[15] J. Lin, E. Keogh, S. Lonardi, and B. Chiu, "A symbolic representation of time series, with implications for streaming algorithms," in Proceedings of ACM SIGMOD workshop on Research issues in data mining and knowledge discovery. ACM, 2003, pp. 2-11.

[16] F. Ganz, P. Barnaghi, and F. Carrez, "Information abstraction for heterogeneous real world internet data," IEEE Sensors Journal, vol. 13, no. 10, pp. 3793-3805, 2013.

[17] S. W. Smith et al., "The scientist and engineer's guide to digital signal processing," 1997.

[18] "LZW data compression: Basics and Implementation,” http://marknelson.us/2011/11/08/lzw-revisited/, Accessed: 2017-03-20.
[19] M. Nati, A. Gluhak, H. Abangar, and W. Headley, "SmartCampus: A user-centric testbed for internet of things experimentation," pp. 1-6, June 2013. 